\title{
Use of opposite frictional forces by animals to increase their attachment reliability during movement
}

\author{
Zhouyi WANG ${ }^{1,2}$, Yi SONG ${ }^{1,3}$, Zhendong DAI ${ }^{1, *}$ \\ ${ }^{1}$ Institute of Bio-inspired Structure and Surface Engineering, Nanjing University of Aeronautics and Astronautics, Nanjing, 210016, China \\ ${ }^{2}$ College of Mechanical and Electrical Engineering, Nanjing University of Aeronautics and Astronautics, Nanjing, Nanjing, 210016, China \\ ${ }^{3}$ College of Aerospace Engineering, Nanjing University of Aeronautics and Astronautics, Nanjing, Nanjing, 210016, China \\ Received: 08 January 2013 / Revised: 04 March 2013 / Accepted: 13 March 2013 \\ C The author(s) 2013. This article is published with open access at Springerlink.com
}

\begin{abstract}
Many animals have the natural ability to move on various surfaces, such as those having different roughness and slope substrates, or even vertical walls and ceilings. Legged animals primarily attach to surfaces using claws, soft and hairy pads, or combinations of them. Recent studies have indicated that the frictional forces generated by these structures not only control the movement of animals but also significantly increase the reliability of their attachment. Moreover, the frictional forces of various animals have opposite characteristics and hierarchical properties from toe-to-toe and leg-to-leg. These opposite frictional forces allow animals to attach securely and stably during movement. The coordination of several attachment (adhesion) modes not only helps animals adhere, which would be impossible in single mode, but also increases the overall stability of the attachment (adhesion) system. These findings can help the design of highly adaptable feet for bionic robots in the near future.
\end{abstract}

Keywords: opposite frictional forces; claw interlocks; soft pad adhesion; hairy pad adhesion; attachment reliability

\section{Introduction}

Motion is a fundamental animal characteristic that defines behavioral traits such as predation, escape, courtship, and reproduction. Although there are numerous legged species with various types of complex movements, all of them require the animals to overcome their body weight, and adapt to complex land morphology and environment. Therefore, legged animals have evolved different morphological structures, topological structures, and movement modes to adapt to their living environment [1] and have developed superb abilities to maintain dynamic stability [2], climb obstacles [3, 4], and achieve shock absorption and antifriction [5, 6]. In particular, many animals move with easiness in a variety of complex surfaces using highly evolved feet. For example, the ability of geckos [7-9], insects [10-14], and spiders

* Corresponding author: Zhendong Dai.

E-mail: Zddai@nuaa.edu.cn
$[15,16]$ to move on different types of surfaces is valuable to bionic design. Moreover, the stability, flexibility, robustness, adaptability, and use of energy displayed by animals are still challenges for bionic robots [17]. Previous studies have indicated that the first obstacle a robot must overcome is the fast and reliable attachment (adhesion) of the robot's feet to the surface. The contact between the robot's feet and the surface should generate frictional or adhesive forces, which the robot uses to move. This study discusses how animals increase their attachment reliability by using opposite frictional forces. Finally, the principle behind the mechanism of opposite frictional forces is introduced into the design of highly adaptable robot soles.

\section{Opposite friction and animal adhesion}

Animals primarily attach to surfaces using claw interlocking, pad adhesion, and setal adhesion; 
opposite frictional forces were found in all these attachment modes.

\subsection{Opposite friction and claw structures}

Animals use claw attachment to balance the gravitational and inertial forces with the help of the frictional force between the chitin-based claws and the surface, which has a microprofile that can be regarded as spheres compared with the animal's claws [18]. The frictional mechanism of a single claw on a spherical surface closely resembles that of a point on a surface with the stable margin located in the friction cone (Fig. 1(a)). The stability of this type of attachment mode depends on the physical properties of the claws and the contact surface. For example, the stability of a beetle's attachment on a rough surface is determined by the friction coefficient of its claws with the corresponding contact surface and the curvature of the contact tips [18] (Fig. 1(a)). Locusts can safely attach on rough ceilings where the diameters of the rough peaks are larger than the radii of the claw tips but they fail when they try to attach on smooth substrates where the diameters of the smooth peaks are equivalent to or smaller than the tips [13]. A single-claw interlock has poor anti-interference ability and can easily become unstable. Thus, most wall-climbing animals have two claws on the terminals of their legs; a feature that enhances their attachment stability (Fig. 1(b)) because of the generated opposite forces [19]. Figures 1(c) and 1(d) show how the opposite frictional forces at two different contact points prevent the claws from lateral slipping, which extends the contact model proposed by Dai et al. [18]. Assuming that the attachment system is laterally self-balanced according to its specific geometrical structure; that is $F_{Q x}=F_{P x}$, the load along the leg axis $F_{x z}$ will strengthen the mechanical locking and result in good contact, when the force angle is smaller than the friction angle, which helps animals attach to rough and inclined surfaces. On the other hand, a small disturbance force against the leg axis will eliminate locking, showing the evident asymmetric character of this attachment mode. Moreover, animals regulate the shared loads on two claws to generate unequal opposite frictional forces and increase the antirollover ability within a safe frictional margin (Fig. 1(d)).
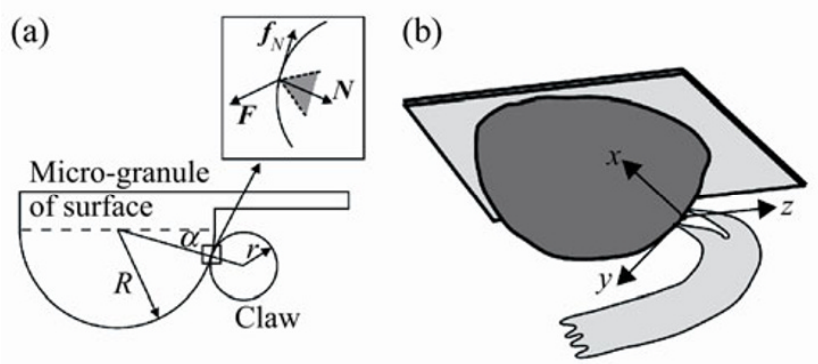

(c)

(d)
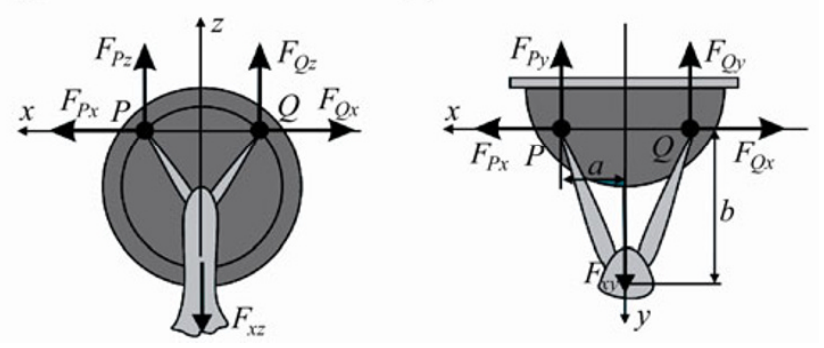

Fig. 1 (a) Model of single claw contact with micro surface particle, where $\alpha$ is the contact angle, $R$ is the radius of the particle, $\boldsymbol{F}$ is the force acting on the claw, $N$ is the normal load, and $\boldsymbol{f}_{N}$ is the tangential force. The shaded area is the frictional cone [18]. (b)-(d) Threedimensional models of an insect claw attaches to a micro surface granule. (The coordinate origin is the midpoint of line between two contact points; $x$ axis is along the line between two contact points; $z$ axis is parallel to the substrate and perpendicular to the $x$ axis; $x$, $y, z$ axis accord with the Cartesian coordinate system.)

\subsection{Opposite friction and soft pad structures}

Animals reduce the impact force during attachment and generate capillary-based adhesion by using soft pads [20]. Dendrocola ants can resist separating forces of 40-150 times their body weight on smooth surfaces. The elastic deformation of oscules also contributes to frictional forces because the adhesive and frictional forces produced by mucus alone are not strong enough to secure the movements of ants as determined by interference reflection microscopy (IRM) analyses and estimates of the thickness and viscosity of the mucous membranes [21]. When Lycorma delicatula specimens contact glass, the contact areas in the tangential contact state are typically larger than those in the normal contact state with frictional and adhesive forces per unit area of $312-900 \mathrm{mN} / \mathrm{mm}^{2}$ and $83-119 \mathrm{mN} / \mathrm{mm}^{2}$, respectively [14]. The deformable epidermis of locust claws has different material and mechanical properties, and microstructure compared with the neighboring epidermis. Such differences lead to different mechanical 
properties [22]. The geometry, structure, material design, and plasma and other internal tissues give locust pads very low contact stiffness (Fig. 2(a)) [20]. As a result, locusts have large contact areas. In locusts, because the directions of the grovy structures on the endosexine of the epidermis of the pads are parallel to the primary cuticula, the grovy structures cannot restrict the deformation of the epidermis in these directions. Hence, flexible pads have large contact areas and adapt to contact surfaces well (Fig. 2(a)) [23]. Consequently, animals create large adhesive forces and opposite frictional forces by increasing their contact areas through the elastic deformation of their pads if pad adhesion is used [20]. In addition, opposite frictional forces significantly contribute to adhesion in this mode. According to finite element method (FEM) results [20] (Fig. 2(b)), the biggest pulling stresses in the entire pad were located at the grovy structures. In addition, the deformation of the contact structures showed that there were lateral displacements at projecting parts during the contact process, indicating that the presence of opposite frictional forces in these parts. Similar to the interlocks of double claws, the scalar sum of $F_{Q y}$ and $F_{P y}$ equals the normal load while their vector sum balances the tangential load (Fig. 2(c)). Thus, the safety margin for adhesion and friction, and the antisideslip ability are enhanced. The difference derives from the fact that the contact areas obviously change depending on the loads because of the special structure of the pads, and these changes help increase the friction coefficient, frictional force, and adhesive stability.

\subsection{Opposite friction and hairy structures}

Flies, geckos, and some beetles have the ability to move on various inclined substrates by using hairy pads and the so-called dry adhesion. Previous studies have shown that the pads of the soles of flies have an elliptical profile and primarily consist of an elastic epidermis. They are covered with setae, which increase the actual adsorption areas [24]. Furthermore, the direction of the setae arrays helps flies control the adhesive and friction forces, and thus generate opposite frictional forces on the right and left pads. Geckos' setae exhibit anisotropic features in opposite directions as well. First, Young's modulus differ along the direction that the setae bend and the opposite direction [25]. Second, the deformation of the setae creates crush and friction forces with the rubbing surfaces, whereas the normal forces obey Coulomb's friction law in the direction opposite to the direction that the setae bend. For preloads, the adhesive and friction forces were measured four times along the bending direction of the setae [26, 27] (Fig. 3(a)). A friction force of about $200 \mu \mathrm{N}$ and a maximum adhesive force of about $40 \mu \mathrm{N}$ were measured for the adhesion of a micron-sized single-sheared seta that detaches around $30^{\circ}$ [28]. The setae arrays and toes of geckos also display asymmetric friction. Moreover, the friction forces along the setae arrays and toes are larger than those in the opposite direction [26]. The angles $\psi$ between the tangential forces on the contact plane and the toes on the vertical walls and ceilings are $12.6^{\circ}$ and $3.1^{\circ}$, respectively, whereas the angles between the reaction forces and motion planes are approximately equal to $20^{\circ}$, thus securing attachment (Fig. 3(b)). The adhesive forces perpendicular to the surfaces are sufficient to balance the animals' weight and the moments caused by weight. The opposite friction forces at the first and fifth toe of the geckos form an interlock on the contact plane, which increases the stability and reliability of the attachment [29] (Fig. 3(c)).
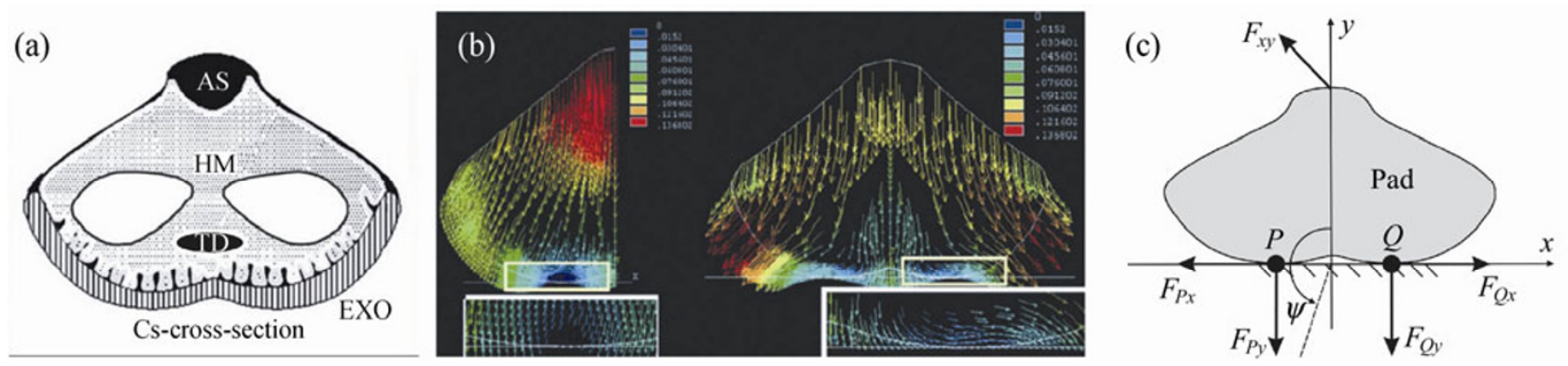

Fig. 2 (a) Cross-sectional structural representation of locust's pad. EXO is the pad epidermis, which contains rod-shaped tissues and appeared to be smooth when observed with a light microscope. (b) Vectorial deformation field of locust pad [20]. (c) Force analysis of locust pad. 

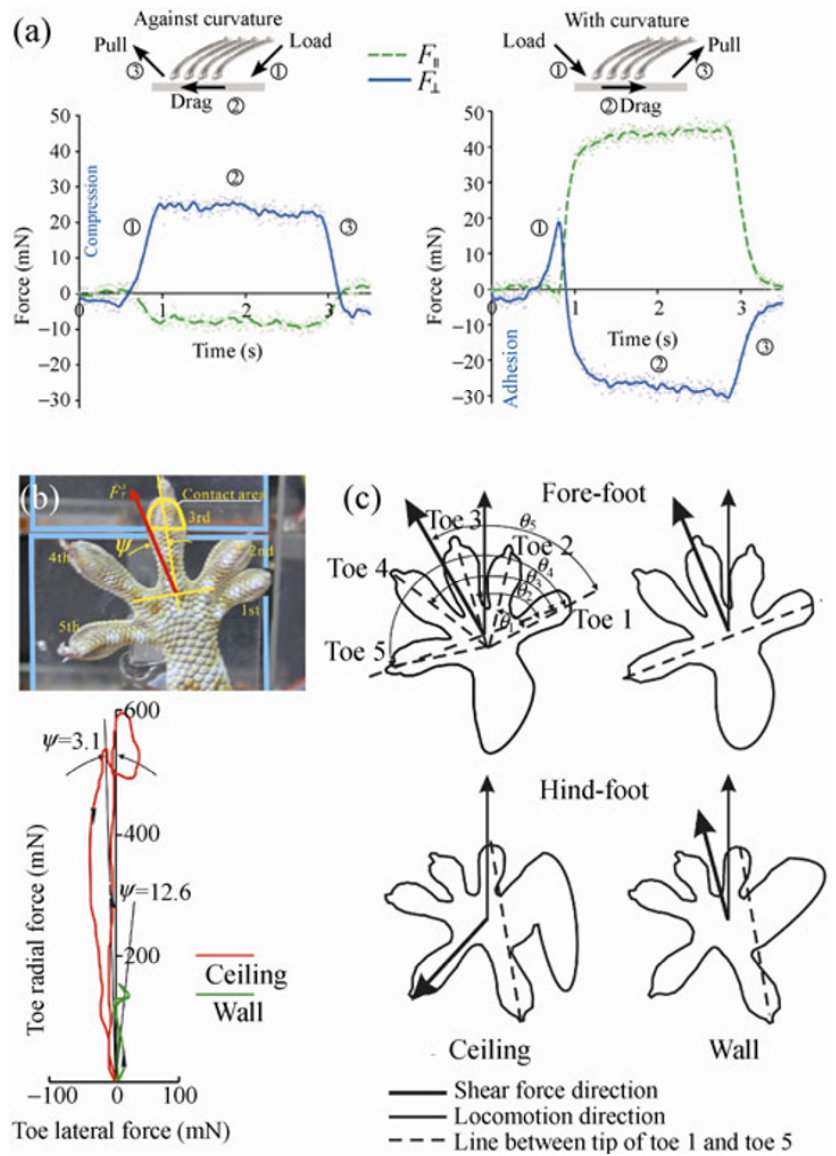

Fig. 3 (a) Experiments on opposite frictions of setae arrays [26]. (b) Measurements of lateral forces of a single gecko toe along its direction [29]. (c) Patterns of gecko sole on ceiling.

\section{Opposite friction between different adhesion modes}

No attachment mode is completely versatile because the physical properties of contact surfaces heavily influence attachment. For example, the stability of the claw interlock is limited by the roughness and friction coefficient of the substrates, and the relative scales of the claws and surface particles [18]. Pad adhesion is highly influenced by the actual microcontact areas, whereas setae adhesions are affected by the actual microcontact angles of the setae [26, 28]. Many animals have more than one tool for attaching on the various substrates. The soles of the toes of geckos are covered with setae even though each toe has a terminal claw; soft pads or setae pads and claws exist concurrently on the tarsal extremities of many insects. Animals make intelligent use of the different adhesion modes of their feet to stably attach to different substrates. Geckos catch surface particles with their claws on rough surfaces while they use their setae to attach to smooth inclined surfaces $[8,26]$. Gampsocleis gratiosa [30] creeps along vertical glass using flexible pads, where the tangential forces (friction forces) are much larger than the normal adhesive forces because they can insert pad cuticles into the microstructures of the glass surface. Locusts, which belong to Orthoptera just like Gampsocleis gratiosa, can reliably grasp the surface particles on sandpaper with microsurface profiles containing spheres of $12-41 \mu \mathrm{m}$ in diameter [21]. Animals can move on surfaces with a roughness comparable to their critical microscale by coordinating the opposite frictions generated by the different attachment modes. Geckos can securely attach to a smooth glass ceiling by overcoming the adhesive angle increment caused by gravity and keep this angle smaller than the critical angle at all times by using the opposite friction forces at their two toes [9, 31, 32] (Fig. 4(a)). Clearly, these findings will increase the operating range of bionic robots.

The coordination of the different attachment modes ensures that the animals have the ability to attach to multiple surfaces. Without considering their internal microstructures and reciprocities, the setae-and-setae
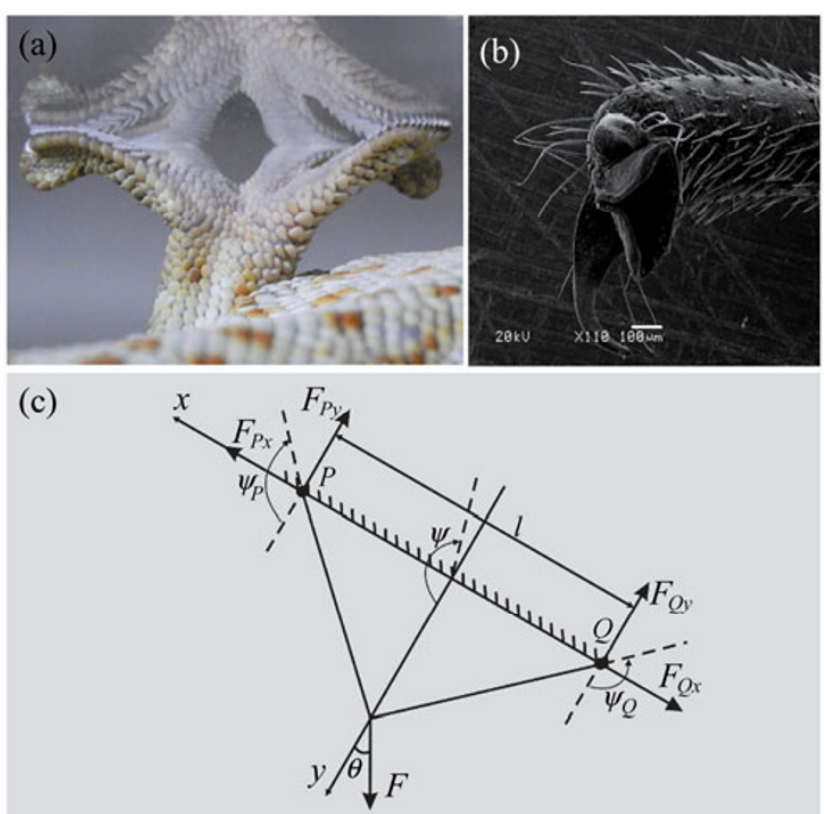

Fig. 4 (a) Pattern of gecko sole on incline. (b) Electron micrograph of Erthesina fullo sole. (c) Mechanical model of coupled attachment modes. 
(Fig. 4(a)) and claw-and-pads (Fig. 4(b)) attachment modes can be simplified to the module shown in Fig. 4(c).

Equivalent frictional angles are introduced for the attachment modes that are different at the two contact areas. The equivalent frictional angle $\psi_{P}$ and $\psi_{Q}$ at points $P$ and $Q$, respectively, satisfies the inequality.

$$
\left\{\begin{array}{l}
F_{Q y} \leqslant F_{Q x} \cdot \tan \left(\pi-\psi_{Q}\right) \\
F_{P x} \leqslant F_{P y} \cdot \tan \left(\pi-\psi_{P}\right)
\end{array}\right.
$$

Hence, the system can be also described by

$$
\tan (\pi-\psi) \leqslant \frac{F_{P y} \cdot \tan \left(\pi-\psi_{P}\right)-F_{Q y} / \tan \left(\pi-\psi_{Q}\right)}{F_{P y}+F_{Q y}}
$$

where $\psi$ is the total equivalent frictional angle and from Formula (2) $\psi>\psi_{Q}$, and $\psi>\psi_{P}$. These two inequalities imply that because of the opposite friction forces, the total equivalent frictional angle of the synergetic attachment modes is larger than that of any single mode, which is the most unique trait of opposite frictional attachment, and increases the attachment safety margin. Combined with earlier findings, it is easy to see that animals form stable triangles on contact planes and stable tetrahedra using the reciprocities of the leg mechanisms and surfaces because the directions of the frictional forces at different contact areas are different. Two of the toes of geckos can exert a couple of opposite frictional forces, whereas all toes and the surface form a tridimensional stable area. The combined attachments not only are more reliable and safer than single-mode attachments but also show high antijamming ability.

In addition, the research regarding the climbing ability of geckos [8], tree frogs [33], and locusts [13] show that their left and right legs in the stance phase need to generate opposite lateral forces, or sometimes opposite shear forces, to increase the stability of the attachment on an inclined surface. This suggests the contribution of opposite forces at different scales, from the basic-level-toe-to-toe in geckos, claw-to-claw in beetles, and left-to-right projections in the soft pads of locusts-to higher-level legs between the left and right side of the animals. Therefore, the movements of animals are processes in which opposite frictional forces operate from the micro- to the macro-level. This means that multiscale opposite frictional forces guarantee the stability and reliability of the locomotion of animals.

\section{Acknowledgments}

This work was supported by the National Natural Science Foundation of China (Grant No. 60910007) and the Fundamental Research Funds for the Central Universities (Grant Nos. CXZZ11_0198 and BCXJ10_10).

Open Access: This article is distributed under the terms of the Creative Commons Attribution Noncommercial License which permits any noncommercial use, distribution, and reproduction in any medium, provided the original author(s) and source are credited.

\section{Reference}

[1] Lu Y X. Significance and progress of bionics. J Bionic Eng 1(1): 1-3 (2004)

[2] Jindrich D L, Full R J. Dynamic stabilization of rapid hexapedal locomotion. J Exp Biol 205: 2803-2823 (2002)

[3] Lammers A R, Earls K D, Biknevicius A R. Locomotor kinetics and kinematics on inclines and declines in the gray short-tailed opossum Monodelphis domestica. J Exp Biol 209: 4154-4166 (2006)

[4] Lammers A R. Locomotor kinetics on sloped arboreal and terrestrial substrates in a small quadrupedal mammal. Zoology 110: 93-103 (2007)

[5] Zhou Q, He B, Qian M G, Yue J G. Analysis on friction and adhesive force of insects pads. J Uni Shanghai Sci Technol 2(30): 143-146 (2008)

[6] Zhou Q, He B, Qian M G, Yue J G. Testing of wet adhesive forces of ants and ANSYS analysis. J Tongji Uni (Nat Sci) 35: 670-673 (2008)

[7] Chen J J, Peattie A M, Autumn K, Full R G. Differential leg function in a sprawled-posture quadrupedal trotter. $J$ Exp Biol 209: 249-259 (2006)

[8] Wang Z Y, Wang J T, Ji A H, Dai Z D. Locomotion behavior and dynamics of geckos freely moving on the ceiling. Chin Sci Bull 55: 3356-3362 (2010)

[9] Pesika N S, Tian Y, Zhao B X, Rosenberg K, Zeng H, McGuiggan P, Israelachvili J N. Peel-zone model of tape peeling based on the gecko adhesive system. $J$ Adhesion $\mathbf{8 3}$ : 383-401 (2007) 
[10] Chen D H, Tong J, Sun J Y, Ren L Q. Tribological behavior of Gampsocleis gratiosa foot pad against vertical flat surfaces. J Bionic Eng 2(4): 187-194 (2005)

[11] Gorb S N. Attachment Devices of Insect Cuticle. Berlin: Kluwer Academic Publishers, 2002.

[12] Gorb S N, Jiao Y, Scherge M. Ultrastructural architecture and mechanical properties of attachment pads in Tettigonia viridissima (Orthoptera Tettigoniidae). J Comp Physiol A 186: 821-831 (2000)

[13] Han L B, Wang Z Y, Ji A H, Dai Z D. Grip and detachment of locusts on inverted sandpaper substrates. Bioinspir Biomim 6: 386-392 (2011)

[14] Frantsevich L, Ji A H, Dai Z D, Wang J, Frantsevich L, Gorb S N. Adhesive properties of the arolium of a lanternfly, Lycorma delicatula (Auchenorrhyncha, Fulgoridae). J Insect Physio 54: 818-827 (2008)

[15] Niederegger S, Gorb S N. Friction and adhesion in the tarsal and metatarsal scopulae of spiders. J Comp Physiol A 192: 1223-1232 (2006)

[16] Wang Z Y, Wang J T, Ji A H, Li H K, Dai Z D. Movement behavior of a spider on a horizontal surface. Chin Sci Bull 56(25): 2748-2757 (2011)

[17] Dickinson M H, Farley C T, Full R J, Koehl M A R, Kram R, Lehman S. How animals move: An Integrative view. Science 288(7): 100-106 (2000)

[18] Dai Z D, Gorb S N and Schwarz U. Roughness-dependent friction force of the tarsal claw system in the beetle Pachnoda marginata (Coleoptera, Scarabaeidae). J Exp Biol 205: 24792488 (2002)

[19] Dai Z D, Yu M, Ji A H. Study on tribological characteristics of animals' driving pads and their bionic design (in Chinese). Chin Mech Eng 8: 1454-1457 (2005)

[20] Dai Z D, Gorb S N. A study on contact mechanics of grasschopper's pad (Insecta: Orthoptera) by finite element methods. Chin Sci Bull 54(4): 549-555 (2009)

[21] Federle W, Riehle M, Curtis A S G, Full R G. An integrative study of insect adhesion: Mechanics and wet adhesion of pretarsal pads in ants. J Integr Comp Biol 42: 1100-11061 (2002)
[22] Jiao Y, Gorb S, Scherge M. Adhesion measured on the attachment pads of tettigonia viridissima (Orthoptera, insecta). $J$ Exp Biol 203(12) : 1887-1895 (2000)

[23] Gorb S. N. The design of the fly adhesive pad: Distal tenent setae are adapted to the delivery of an adhesive secretion. Proc R Soc Lond B 265: 747-752 (1998)

[24] Eisner T, Aneshansley D J. Defense by foot adhesion in a beetle (Hemisphaerota cyanea). PNAS 97(12): 6568-6573 (2000)

[25] Autumn K, Majidi C, Groff R E, Dittmore A, Fearing R. Effective elastice modulus of isolated gecko setal arrays. J Exp Biol 209: 3558-3568 (2006)

[26] Autumn K, Dittmore A, Santos D, Spenko M, Cutkosky M. Frictional adhesion: A new angle on gecko attachment. $J$ Exp Biol 209: 3569-3579 (2006)

[27] Zhao B X, Pesika N, Rosenberg K, Tian Y, Zeng H, McGuiggan P, Autumn K, Israelachvili J. Adhesion and friction force coupling of gecko setal arrays: Implication for structured adhesive surfaces. Langmuir 24: 1517-1524 (2008)

[28] Autumn K, Liang Y A, Hsieh S T, Zesch W, Chan W P, Kenny T W, Fearing R, Full R J. Adhesive force of a single gecko foot-hair. Nature 405: 681-684 (2000)

[29] Wang Z Y, Gu W H, Wu Q, Ji A H, Dai Z D. Morphology and reaction force of toes of geckos freely moving on ceilings and walls. Sci China Technol Sci 53: 1688-1693 (2010)

[30] Chen D H, Tong J, Sun J Y, Ren L Q. Tribological behavior of gampsocleis Gratiosa foot pad against vertical flat surfaces. J Bionic Eng 2: 187-194 (2005)

[31] Autumn K, Hsieh S T, Dudek D M, Chen J, Chitaphan C, Full R J. Dynamics of geckos running vertically. J Exp Biol 209: 260-272 (2006)

[32] Wang Z Y, Wang J T, Ji A H, Zhang Y Y, Dai Z D. Behavior and dynamics of gecko locomotion: The effects of moving directions on vertical surface. Chin Sci Bull 56: 573-583 (2010)

[33] Endlein T, Ji A H, Samuel D, Yao N, Wang Z H, Barnes W J, Federle W, Kappl M, Dai Z D. Sticking like sticky tape: Tree frogs use friction forces to enhance attachment on overhanging surfaces. J R Soc Interface 10: 1742 (2013) 


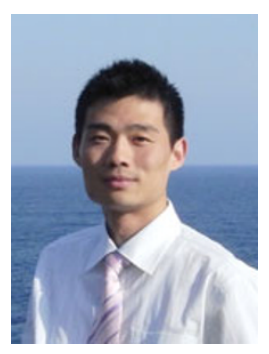

Zhouyi WANG Doctor, obtained his Master degree in 2009 from Nanjing University of Aeronautics and Astronautics (NUAA). He studied as a $\mathrm{PhD}$ Candidate since 2009 at Institute of Bio-inspired Structure

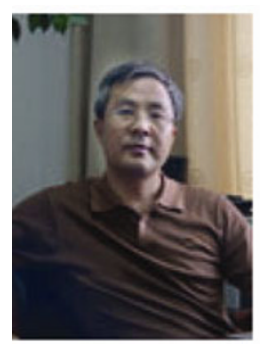

Zhendong DAI Professor and tutor of PhD students, obtained his doctor degree in 1999 from College of Mechanical and Electrical Engineering, NUAA. He is one of the Chinese delegates of International Institute of Bionic Engineering, an executive member of the council of Chinese Mechanical Engineering in Tribology, and a member of the academic committee of State Key Laboratory of Solid Lubrication. He also is a member of editorial board of many academic journals such as Journal and Surface Engineering, NUAA. His interested research areas include tribology, bionics, animal kinematics and dynamics. He has participated in many research projects and has published 12 papers on international journals.

of Bionic Engineering, International Journal of Vehicle Autonomous System, Tribology and so on. He was invited to attend the Advisory Seminar about the development planning of American science foundation and invited to give lectures in Case Western Reserve University, UC San Diego, GIT, Kyoto University, Yonsei University, Cambridge University in 2010. His research areas include bionics, light material, control of bionics, bio-robots, and biological robots. He has successively presided and participated in many research projects and has published more than 200 papers and gotten more than 15 patents. 